\title{
Prompt fission $\gamma$-ray data from spontaneous fission and the mechanism of fission-fragment de-excitation
}

\author{
Stephan Oberstedt ${ }^{1, \mathrm{a}}$, Aleksandar Dragic ${ }^{1,2}$, Angelique Gatera ${ }^{1, \mathrm{~b}}$, Alf Göök ${ }^{1}$, Franz-Josef Hambsch ${ }^{1}$, \\ and Andreas Oberstedt ${ }^{3}$ \\ ${ }^{1}$ European Commission, Joint Research Centre, Directorate for Nuclear Safety and Security, Unit G.2 Standards for Nuclear Safety, \\ Security and Safeguards, 2440 Geel, Belgium \\ 2 Institute of Physics, Pregrevica 118, 11080 Belgrade, Serbia \\ ${ }^{3}$ Extreme Light Infrastructure - Nuclear Physics (ELI-NP) / Horia Hulubei National Institute for Physics and Nuclear Engineering \\ (IFIN-HH), Bucharest-Magurele 077125, Romania
}

\begin{abstract}
The investigation of prompt $\gamma$-ray emission in nuclear fission has a great relevance for the assessment of prompt heat generation in a reactor core and for the better understanding of the de-excitation mechanism of fission fragments. Some years ago experimental data was scarce and available only from a few fission reactions, ${ }^{233,235} \mathrm{U}\left(\mathrm{n}_{t h}, \mathrm{f}\right),{ }^{239} \mathrm{Pu}\left(\mathrm{n}_{t h}, \mathrm{f}\right)$, and ${ }^{252} \mathrm{Cf}(\mathrm{sf})$. Initiated by a high priority data request published by the OECD/NEA a dedicated prompt fission $\gamma$-ray measurement program is being conducted at the Joint Research Centre Geel. In recent years we obtained new and accurate prompt fission $\gamma$-ray spectrum (PFGS) characteristics (average number of photons per fission, average total energy per fission and mean photon energy) from ${ }^{252} \mathrm{Cf}(\mathrm{sf}),{ }^{235} \mathrm{U}\left(\mathrm{n}_{t h}, \mathrm{f}\right)$ and ${ }^{239,241} \mathrm{Pu}\left(\mathrm{n}_{t h}, \mathrm{f}\right)$ within $2 \%$ of uncertainty. In order to understand the dependence of prompt fission $\gamma$-ray emission on the compound nuclear mass and excitation energy, we started a first measurement campaign on spontaneously fissioning plutonium and curium isotopes. Results on PFGS characteristics from ${ }^{240,242} \mathrm{Pu}$ (sf) show a dependence on the fragment mass distribution rather than on the average prompt neutron multiplicity, pointing to a more complex competition between prompt fission $\gamma$-ray and neutron emission.
\end{abstract}

\section{Introduction}

Some years ago new interest was shown in the measurement of prompt fission $\gamma$-ray spectra (PFGS). This was motivated by requests for new precise values especially for $\gamma$-ray multiplicities and average photon energy release per fission in the thermal-neutron induced fission on ${ }^{235} \mathrm{U}$ [1] and ${ }^{239} \mathrm{Pu}$ [2]. Improvements of nuclear data have now become possible due to advances in scintillator materials, as used e.g. in lanthanide halide detectors. They offer a superior combination of intrinsic peak efficiency, energy and timing resolution, as already demonstrated in a number of recent experiments on ${ }^{252} \mathrm{Cf}(\mathrm{sf})[3-5],{ }^{235} \mathrm{U}\left(\mathrm{n}_{t h}, \mathrm{f}\right)[4,6]$ and ${ }^{241} \mathrm{Pu}\left(\mathrm{n}_{t h}, \mathrm{f}\right)[4,7]$.

A first parametrization to describe systematic trends of PFGS characteristics was made by T.E. Valentine [9], where he introduced a somewhat arbitrary functional dependence of PFGS characteristics on the compound nucleus' mass and atomic numbers as well as its prompt fission neutron multiplicity to account for the competition between the two de-excitation channels of prompt $\gamma$-ray and neutron emission. In this early work only ${ }^{252} \mathrm{Cf}(\mathrm{sf})$ and thermal-neutron induced fission were considered. The parameters obtained back then need an adjustment due to recently published experimental results as shown in Refs. [10,11], and JRC Geel started measurements on

\footnotetext{
a e-mail: stephan.oberstedt@ec.europa.eu

$\mathrm{b}$ The European Commission is gratefully acknowledged for providing a $\mathrm{PhD}$ fellowship to one of the authors (A. Gatera).
}

spontaneously fissioning actinides other than ${ }^{252} \mathrm{Cf}$. We obtained first results on PFGS characteristics from the spontaneous fission of ${ }^{240} \mathrm{Pu}$ and ${ }^{242} \mathrm{Pu}$ [8].

\section{Experiment and data treatment}

The prompt fission $\gamma$-ray measurements on ${ }^{240} \mathrm{Pu}$ and ${ }^{242} \mathrm{Pu}$ formed part of a series of experiments, which originally aimed at the precise measurement of their neutron-induced fission cross-sections, in response to a high-priority request published through the OECD/NEA $[12,13]$. In preparation of these measurements a precise determination of the spontaneous fission half-life of each isotope was achieved [14]. For this proceedings we will summarize briefly the experimental setup and the achieved results. More details may be found in Ref. [8].

A pre-requisite for such measurements is the availability of high-quality targets with a high isotopic purity. The preparation of such targets was achieved at the ECJRC Geel [15]. The plutonium targets had an isotopic purity of 99.89 and $99.96 \%$. Thin targets of ${ }^{240} \mathrm{Pu}(92.94$ $\mu \mathrm{g})$ and ${ }^{242} \mathrm{Pu}(6716 \mu \mathrm{g})$ were placed back-to-back at the central cathode position inside a cylindrical twin Frischgrid ionization chamber. The fission rates were $0.044 / \mathrm{s}$ and $0.54 / \mathrm{s}$ for ${ }^{240} \mathrm{Pu}$ and ${ }^{242} \mathrm{Pu}$, respectively. In coincidence with the fission fragments $\gamma$-rays were measured with one coaxial $\mathrm{LaBr}_{3}$ :Ce scintillation detector of size $76.2 \mathrm{~mm} \times$ $76.2 \mathrm{~mm}$ (diameter $\times$ length). 
Table 1. Average prompt fission $\gamma$-ray spectrum characteristics for the spontaneous fission of ${ }^{240} \mathrm{Pu}$ and of ${ }^{242} \mathrm{Pu}$ from this work compared with corresponding results from thermal-neutron induced fission on ${ }^{239,241} \mathrm{Pu}$ from Refs. [7,22] and from systematic trends established in Ref. [9].

\begin{tabular}{lccccc}
\hline \hline$\left({ }^{A} \mathrm{X}\right)_{C N}$ & $\begin{array}{c}\Delta \mathrm{t} \\
(\mathrm{ns})\end{array}$ & $\begin{array}{c}\bar{M}_{\gamma} \\
\text { (/fission) }\end{array}$ & $\begin{array}{c}\bar{\epsilon}_{\gamma} \\
(\mathrm{MeV})\end{array}$ & $\begin{array}{c}\bar{E}_{\gamma, \text { tot }} \\
(\mathrm{MeV})\end{array}$ & Reference \\
\hline${ }^{242} \mathrm{Pu}$ & \pm 10 & $6.72 \pm 0.07$ & $0.843 \pm 0.012$ & $5.66 \pm 0.06$ & this work \\
${ }^{242} \mathrm{Pu}$ & & $6.5 \pm 0.5$ & $0.93 \pm 0.07$ & $6.05 \pm 0.03$ & {$[9]$} \\
${ }^{242} \mathrm{Pu}^{*}$ & \pm 3 & $8.21 \pm 0.09$ & $0.78 \pm 0.01$ & $6.41 \pm 0.06$ & {$[7]$} \\
\hline${ }^{240} \mathrm{Pu}$ & \pm 10 & $8.2 \pm 0.4$ & $0.80 \pm 0.07$ & $6.6 \pm 0.5$ & this work \\
${ }^{240} \mathrm{Pu}$ & & $6.4 \pm 0.5$ & $0.95 \pm 0.07$ & $6.07 \pm 0.03$ & {$[9]$} \\
${ }^{240} \mathrm{Pu}^{*}$ & \pm 10 & 7.23 & $0.94 \pm 0.05$ & $6.81 \pm 0.3$ & {$[22]$} \\
\hline \hline
\end{tabular}

The pulse-height of the $\gamma$-rays was calibrated with standard radioactive sources with decay energies between $81 \mathrm{keV}$ and $4.44 \mathrm{MeV}$, using ${ }^{133} \mathrm{Ba}(81 \mathrm{keV}-356 \mathrm{keV})$, ${ }^{137} \mathrm{Cs} \quad(662 \mathrm{keV}),{ }^{60} \mathrm{Co} \quad(1173 \mathrm{keV}, \quad 1333 \mathrm{keV}),{ }^{232} \mathrm{Th}$ $(583 \mathrm{keV}$ and $2614 \mathrm{keV}$ ) and AmBe (decay of the first excited state in $\left.{ }^{12} \mathrm{C} ; \mathrm{E}_{\gamma}=4.44 \mathrm{MeV}\right)$. The low-energy threshold during the PFGS measurements was set to $100 \mathrm{keV}$.

Due to the DAQ the timing resolution was about $7 \mathrm{~ns}$ (FWHM). This was still sufficient to discriminate prompt fission $\gamma$-rays from photons created in (n, n') reactions of fission neutrons by their time-of-flight relative to the instant of a fission event. With the $\gamma$-ray detector placed at a distance of $4082 \mathrm{~mm}$ relative to the fission source all neutrons with energies below $6.5 \mathrm{MeV}$ were discriminated. The corresponding background-corrected numbers of prompt fission $\gamma$-rays measured in coincidence with a fission fragment were 888 and 8210 .

\section{PFGS characteristics from ${ }^{242} \mathrm{Pu}(\mathrm{sf})$}

To extract an emission spectrum from the measured one, the response function of the detector must be determined and unfolded. The usual procedure is to simulate, by means of Monte-Carlo simulations, the response of the detector to mono-energetic $\gamma$-rays accounting for the geometrical efficiency and the experimental set-up. The resulting spectra are then adjusted to the measured spectrum (for details see Ref. [3]). Due to the limited number of events at energies above $3 \mathrm{MeV}$, an exponential was fitted to the experimental data above $1.8 \mathrm{MeV}$. When extracting the spectral characteristics the uncertainty of the coefficients of the exponential function were properly taken into account.

The resulting prompt fission $\gamma$-ray spectrum is depicted in Fig. 1 by the red line. For comparison we show the emission spectrum obtained previously for the thermalneutron induced fission on ${ }^{241} \mathrm{Pu}$ [7]. Both spectra agree very well with each other. The effect of the extra excitation energy in neutron-induced fission seems to be reflected in a surplus of photons at energies below $500 \mathrm{keV}$. The spectral characteristics, integrated from $0.1 \mathrm{MeV} \leq \mathrm{E}_{\gamma} \leq 7 \mathrm{MeV}$, are summarized in Table 1.

\section{PFGS characteristics from ${ }^{240} \mathrm{Pu}(\mathrm{sf})$}

In the case of ${ }^{242} \mathrm{Pu}(\mathrm{sf})$ we were able to perform the standard unfolding of the measured PFGS. This was not possible in the present study in the case of ${ }^{240} \mathrm{Pu}(\mathrm{sf})$ due to the poor counting statistics of less than 1000 events). Instead we followed here a different approach, based upon

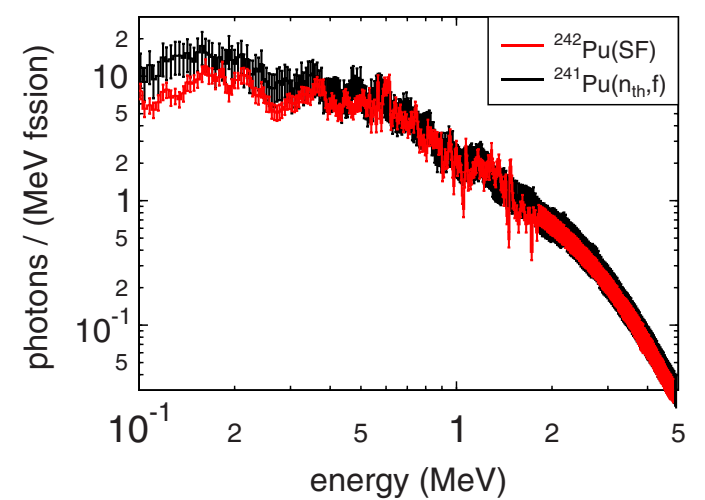

Figure 1. Prompt $\gamma$-ray spectrum for ${ }^{242} \mathrm{Pu}$ (sf) in photons per $\mathrm{MeV}$ and per fission (red line); the corresponding spectrum for ${ }^{241} \mathrm{Pu}\left(\mathrm{n}_{t h}, \mathrm{f}\right)$ [7] is shown for comparison (black line). The data are depicted in logarithmic energy scale to emphasize the lowenergy region.

the definition of a so-called transformation function as detailed in the following.

The method of applying a transformation function is motivated by the fact that we measured both spectral data from two samples in essentially the same position relative to the $\gamma$-detector. Therefore, the response should be identical in both cases reported here. In addition, both measured spectra have to exhibit a very similar shape as we may observe in the case of ${ }^{242} \mathrm{Pu}$ (sf) when compared to that from ${ }^{241} \mathrm{Pu}\left(\mathrm{n}_{t h}, \mathrm{f}\right)$. Hence, we define a transformation function as ratio between the emission spectrum and the measured spectrum:

$$
E S\left(E_{\gamma, i}\right)=T F\left(E_{\gamma, i}\right) \times M S\left(E_{\gamma, i}\right) / N_{\text {fission }} .
$$

Here, $E S\left(E_{\gamma, i}\right)$ denotes the emission spectrum, $M S\left(E_{\gamma, i}\right)$ the measured one and $T F\left(E_{\gamma, i}\right)$ a transformation function. The latter was determined from the experiment on ${ }^{242} \mathrm{Pu}(\mathrm{sf})$. The energies $E_{\gamma, i}$, for which $T F\left(E_{\gamma, i}\right)$ was calculated, correspond to the energies of the unfolded spectrum, to which the measured spectrum was interpolated to by using the Aitken-Neville method [18]. The transformation function contains both the statistical uncertainty from the measured spectrum as well as the uncertainties of the emission spectrum from the unfolding of the response function.

Prior to applying this transformation function to the measured prompt fission $\gamma$-ray spectrum from this work, new values and their uncertainties for matching energies were interpolated again. Finally, we obtain an emission spectrum as depicted as full (blue) line in Fig. 2. The error bars include the statistical uncertainties from the 


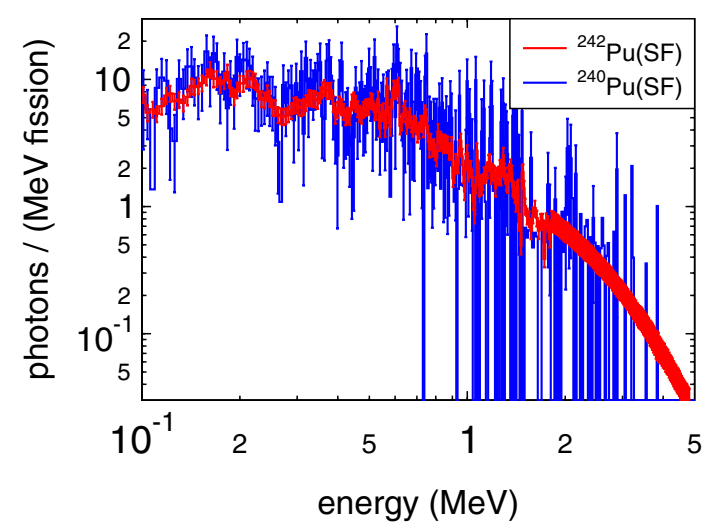

Figure 2. Prompt $\gamma$-ray spectra for ${ }^{240} \mathrm{Pu}(\mathrm{sf})$ and ${ }^{242} \mathrm{Pu}(\mathrm{sf})$ from this work depicted as full red and blue lines, respectively. The data are shown in logarithmic energy scale to emphasize the lowenergy region.

measured spectrum and uncertainties in the transformation function. For comparison the PFGS for ${ }^{242} \mathrm{Pu}(\mathrm{sf})$ is shown as full (red) line. The spectral characteristics, integrated from $0.1 \mathrm{MeV} \leq \mathrm{E}_{\gamma} \leq 4 \mathrm{MeV}$, are summarized in Table 1 . In Fig. 2 we show the spectra with a logarithmic energy scale to provide a better view on the low-energy part below $1 \mathrm{MeV}$. The excellent agreement between both emission spectra is obvious, which implies that also the measured spectra have the same shape indeed and, hence, one of the conditions for the meaningful application of a transformation function is fulfilled.

\section{Results and discussion}

Since we present here the first prompt fission $\gamma$-ray data from spontaneously fissioning ${ }^{240} \mathrm{Pu}$ and ${ }^{242} \mathrm{Pu}$, we compare our results in Table 1 with spectral characteristics drawn from the above mentioned trend established by T.E. Valentine [9]. For a detailed decomposition of the uncertainties we refer to Ref. [8].

In the case of ${ }^{242} \mathrm{Pu}(\mathrm{sf})$ the agreement is very reasonable, taking into account that the trend is based on a few experimental data available back then. The mean energy per photon, $\epsilon_{\gamma}$, is close to $0.8 \mathrm{MeV}$. From our previous measurements we have evidence that the average energy per photon shows only weak dependence on the compound system and even on the incident neutron energy. That the multiplicity is somewhat higher, is in line with our findings in ${ }^{252} \mathrm{Cf}(\mathrm{sf}) \quad[3-5],{ }^{235} \mathrm{U}\left(\mathrm{n}_{t h}, \mathrm{f}\right) \quad[4,6]$ and ${ }^{241} \mathrm{Pu}\left(\mathrm{n}_{t h}, \mathrm{f}\right)$ $[4,7]$. This is understandable when remembering that the low-energy threshold in our measurements is in most cases lower than in measurements conducted in the past. The different average multiplicity, $\bar{M}_{\gamma}$, observed between spontaneous and neutron-induced fission of ${ }^{242} \mathrm{Pu}$ scales well with the average total prompt neutron multiplicity for both fissioning systems, $\bar{v}_{s f}=2.134(6)$ and $\bar{v}_{t h}=2.946(6)$ [19]. The influence of the different widths of the prompt time window, $\Delta \mathrm{t}$ (cf. Table 1), may be neglected, because $\Delta \mathrm{t} \geq 1.43 F W H M_{t}$ in both cases.

In case of ${ }^{240} \mathrm{Pu}(\mathrm{sf}), \bar{M}_{\gamma}$ appears on first sight pretty high, definitely when comparing with the predictions drawn from Valentine's trend. The differences may be regarded as significant despite the much larger uncertainty. However, our value for the average energy per photon,

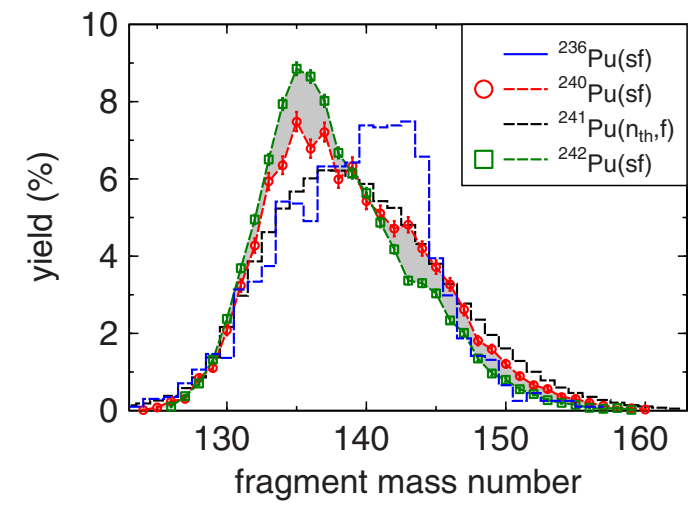

Figure 3. Pre-neutron fission-fragment yield distribution from ${ }^{236,240,242} \mathrm{Pu}(\mathrm{sf})$ and ${ }^{241} \mathrm{Pu}\left(\mathrm{n}_{t h}, \mathrm{f}\right)$ in the heavy fragment mass region [20].

$\epsilon_{\gamma}=0.8 \mathrm{MeV}$, is well in agreement with all our other results, providing us with confidence in our new PFGS data for ${ }^{240} \mathrm{Pu}(\mathrm{sf})$ as well as in the underlying data analysis.

Systematic measurements of pre-neutron mass distributions from different spontaneously fissioning plutonium isotopes have revealed significant changes in fission fragment yield distribution as a function of neutron number of the compound nucleus [20]. Starting from ${ }^{236} \mathrm{Pu}$, the maximum yield of heavy fragments, $\mathrm{A}_{h}$, is around $142 \mathrm{u}$ (Fig. 3). This position moves down to $\mathrm{A}_{h} \approx 135 \mathrm{u}$ for ${ }^{242} \mathrm{Pu}$, i.e. increasing the fraction of less-deformed fragments being close to the doubly magic isotope ${ }^{132} \mathrm{Sn}$. One might, therefore, argue that in case of ${ }^{240} \mathrm{Pu}(\mathrm{sf})$ the dominant fraction of deformed fragments leads to an enhanced multiplicity due to higher level densities manifesting in a higher $\bar{M}_{\gamma}$.

However, one must not forget that for both spontaneously fissioning isotopes $\bar{v}_{s f}$ is essentially the same with $\delta \bar{v}_{s f}\left({ }^{240} \mathrm{Pu},{ }^{242} \mathrm{Pu}\right)<0.4 \%$ [19]. In Ref. [20] we find that the average total kinetic energy, $\overline{T K E}$, is lower for ${ }^{240} \mathrm{Pu}(\mathrm{sf})$ by almost $2 \mathrm{MeV}$. This small amount of more excitation energy, depending on the Q-value of the reaction, does not seem to lead to an enhanced average neutron multiplicity, but may account for the excess observed for $\bar{M}_{\gamma}$.

\section{Conclusion}

PFGS characteristics from the spontaneous fission of ${ }^{240} \mathrm{Pu}$ and of ${ }^{242} \mathrm{Pu}$ were measured for the first time with reasonable statistical accuracy. This was possible in the case of ${ }^{240} \mathrm{Pu}$ by applying a transformation function on the measured spectrum in order to deduce the emission spectrum from a measured spectrum instead of a proper unfolding procedure by a response function. The transformation function was obtained from ${ }^{242} \mathrm{Pu}(\mathrm{sf})$ measured with sufficient statistics under exactly the same conditions. This procedure seems to work well as long as reference spectra exist, that have a very similar shape, which is the case here. Hence, this technique should be considered as an alternative, if recorded spectra are obtained from a low number of events (see Ref. [8] and Refs. therein).

The results presented in this work fit well into the trend established by Valentine [9] and recently revised 
in Refs. $[10,11]$. They may be regarded as the first step to enlarge the data base from which new systematic trends as a function of incident neutron energy are established.

The significantly enhanced average multiplicity in case of ${ }^{240} \mathrm{Pu}(\mathrm{sf})$ points to a possible correlation of prompt $\gamma$-ray emission with the distinct shape of the fission fragment distribution. To fully understand the underlying physics more data is needed. Exchange with theoreticians is highly encouraged. In particular PFGS measurements on ${ }^{244} \mathrm{Pu}(\mathrm{sf})$ and different spontaneously fissioning curium isotopes are recommended. For the latter also measurements of pre-neutron fragment distributions Y(A, TKE) will be indispensable.

A PFGS measurement on the reaction ${ }^{239} \mathrm{Pu}\left(\mathrm{n}_{t h}, \mathrm{f}\right)$ to be compared with ${ }^{240} \mathrm{Pu}(\mathrm{sf})$ in analogy to the presented ${ }^{242} \mathrm{Pu}$ case is subject of an on-going campaign.

\section{References}

[1] Nuclear Data High Priority Request List of the NEA (Req. ID: H.4), www . oecd-nea.org/dbdata/ hprl/hprlview.pl?ID=422

[2] Nuclear Data High Priority Request List of the NEA (Req. ID: H.3), www . oecd-nea.org/dbdata/ hprl/hprlview . pl? ID $=421$

[3] R. Billnert, F.-J. Hambsch, A. Oberstedt, and S. Oberstedt, Phys. Rev. C 87, 024601 (2013)

[4] R. Billnert, T. Belgya, T. Bryś, W. Geerts, C. Guerrero, F.-J. Hambsch, Z. Kis, A. Oberstedt, S. Oberstedt, L. Szentmiklosi, K. Takàcs, and M. Vidali, Phys. Proc. 59, 17 (2014)

[5] A. Oberstedt, R. Billnert, F.-J. Hambsch, and S. Oberstedt, Phys. Rev. C 92, 014618 (2015)

[6] A. Oberstedt, T. Belgya, R. Billnert, R. Borcea, T. Bryś, W. Geerts, A. Göök, F.-J. Hambsch, Z. Kis, T. Martinez, S. Oberstedt, L. Szentmiklosi, K. Takàcs, and M. Vidali, Phys. Rev. C 87, 051602(R) (2013)

[7] S. Oberstedt, R. Billnert, T. Belgya, T. Bryś, W. Geerts, C. Guerrero, F.-J. Hambsch, Z. Kis, A. Moens, A. Oberstedt, G. Sibbens, L. Szentmiklosi, D. Vanleeuw, and M. Vidali, Phys. Rev. C 90, 024618 (2014)
[8] S. Oberstedt, A. Gatera, A. Göök, F.-J. Hambsch and A Oberstedt, Phys. Rev. C 93, 054603 (2016)

[9] T.E. Valentine, Ann. Nucl. Energy 28, 191 (2001)

[10] A. Oberstedt, R. Billnert and S. Oberstedt, Proceedings of NEMEA-7 The $7^{\text {th }}$ Workshop on Nuclear Measurements, Evaluations and Applications, November 5-8, 2013, Geel, Belgium, OECD Nuclear Science NEA/NSC/DOC 13, 199 (2014)

[11] A. Oberstedt, R. Billnert, and S. Oberstedt, manuscript to be submitted

[12] Nuclear Data High Priority Request List of the NEA (Req. ID: H.37), www. oecd-nea.org/dbdata/ hprl/hprlview . pl? ID $=457$

[13] Nuclear Data High Priority Request List of the NEA (Req. ID: H.39), www.oecd-nea.org/dbdata/ hprl/hprlview $\cdot \mathrm{pl}$ ? ID $=457$

[14] P. Salvador-Castiñeira, T. Brys̀, R. Eykens, F.-J. Hambsch, A. Moens, S. Oberstedt, G. Sibbens, D. Vanleeuw, and M. Vidali, Phys. Rev. C 88, 064611 (2013)

[15] G. Sibbens, A. Moens, R. Eykens, D. Vanleeuw, F. Kehoe, H. Kühn, R. Jakopic, S. Richter, A. Plompen, Y. Aregbe, J. Radioanal. Nucl. Chem. 299, 1093-1098 (2014)

[16] www.mesytec.com/datasheets/MPR-1.pdf

[17] http://www.keysight.com/en/pd-1184897pn-U1065A/acqiris-10-bit-high-speedcpci-digitizers?\&cc $=B E \& l c=d u t$

[18] I.N. Bronstein, K.A. Semendjajew, G. Musiol, H. Mühlig, Taschenbuch der Mathematik mit Multiplattform-CD-ROM, 5. Edition, Verlag Harri Deutsch, Frankfurt am Main, ISBN: 978-3-81712017-8 (2000)

[19] F. Gönnenwein, in: C. Wagemans (editor), The Nuclear Fission Process, CRC press (1991), ISBN 0-8493-5443-X, Chapter 11, 514

[20] L. Demattè, C. Wagemans, R. Barthelemy, P. D'Hondt, A. Deruytter, Nucl. Phys. A 614, 331 (1997)

[21] R. Gold, An Iterative Unfolding Method for Response Matrices, Argonne National Laboratory, ANL-6984 (1964)

[22] V.V. Verbinski, Hans Weber, and R.E: Sund, Phys. Rev. C 7, 1173 (1973) 